PROCEEDINGS OF THE

AMERICAN MATHEMATICAL SOCIETY

Volume 131, Number 5, Pages 1329-1338

S 0002-9939(02)06972-1

Article electronically published on December 6, 2002

\title{
ALGEBRAS WITHOUT NOETHERIAN FILTRATIONS
}

\author{
J. T. STAFFORD AND J. J. ZHANG
}

(Communicated by Lance W. Small)

\begin{abstract}
We provide examples of finitely generated noetherian PI algebras for which there is no finite dimensional filtration with a noetherian associated graded ring; thus we answer negatively a question of Lorenz (1988).
\end{abstract}

\section{INTRODUCTION}

In this paper all algebras will be defined over a fixed base field $k$. Let $\Gamma$ be an $\mathbb{N}$-filtration of a $k$-algebra $A$; thus, $\Gamma=\left\{\Gamma_{i}: i \in \mathbb{N}\right\}$ is an ascending chain of $k$-subspaces of $A$ satisfying $1 \in \Gamma_{0}, \bigcup_{j \in \mathbb{N}} \Gamma_{j}=A$ and $\Gamma_{i} \Gamma_{j} \subseteq \Gamma_{i+j}$ for all $i, j$. The filtration is finite if $\operatorname{dim}_{k} \Gamma_{i}<\infty$ for all $i \in \mathbb{N}$ and standard if $\Gamma_{0}=k$ and $\Gamma_{i}=\Gamma_{1}^{i}$ for all $i \geq 2$. We say that $\Gamma$ is a (left) noetherian filtration if the associated graded ring gr $A=\operatorname{gr}_{\Gamma} A=\bigoplus_{i} \Gamma_{i} / \Gamma_{i-1}$ is (left) noetherian. The algebra $A$ is affine if it is finitely generated as a $k$-algebra.

If an algebra $A$ has a left noetherian $\mathbb{N}$-filtration, then a standard technique is to pull results back from gr $A$ to $A$ since theorems are typically easier to prove in the graded ring gr $A$ than in $A$. For related reasons Lorenz asked in [6, p. 436] and [7, Question III.4.2] whether every left noetherian affine algebra $R$, satisfying a polynomial identity (PI), admits a left noetherian, standard finite $\mathbb{N}$-filtration. This question has been raised again (without the "standard" hypothesis) in [15. Question 6.16] because of its importance for dualizing complexes and homological questions: if Lorenz's question were to have a positive answer, then $R$ would have a dualizing complex [15. Corollary 6.9] and every noetherian affine PI Hopf algebra would have finite injective dimension [13].

The aim of this note is to answer these questions by providing a class of noetherian affine PI algebras which do not admit any noetherian finite $\mathbb{N}$-filtration. The basic technique is provided by the following theorem (see Section 21).

Theorem 1.1. Suppose that $I$ and $J_{1} \subset J_{2}$ are ideals of an algebra $R$ such that $J_{2} / J_{1}$ is free of rank $s$ as a left $R / I$-module and free of rank $t$ as a right $R / I$ module. If $s<t$, then there is no finite $\mathbb{N}$-filtration $\Gamma$ of $R$ such that $\operatorname{gr}_{\Gamma} R$ is left noetherian.

Received by the editors September 25, 2000.

2000 Mathematics Subject Classification. Primary 16P40, 16P90, 16R99, 16 W70.

Key words and phrases. PI algebra, noetherian filtration, associated graded ring.

Both authors were supported in part by the NSF. The second author was also supported by the Royalty Research Fund of the University of Washington.

(C)2002 American Mathematical Society 
A variant of this theorem also holds if one replaces "free of rank $x$ " by "of Goldie rank $x$." See Theorem 3.2 for the details.

A simple example satisfying the hypotheses (and conclusion) of the theorem is given by the ring

$$
R=\left\{\left(\begin{array}{cc}
f(x) & g(x) \\
0 & f\left(x^{2}\right)
\end{array}\right): f, g \in k[x]\right\} \subset M_{2}(k[x]) .
$$

Here, one takes $J_{2}=I$ to be the ideal of strictly upper triangular matrices and $J_{1}=0$. See Section 4 for more details.

The most important case of Theorem 1.1 is when $\Gamma$ is a standard. However, we emphasize that the theorem holds for any filtration satisfying the earlier definitions.

The analogue of Theorem 1.1 also holds for $\mathfrak{m}$-adic filtrations (where $\mathfrak{m}$ is the Jacobson radical of a local algebra) and for that reason we prove the result for rings with a Zariskian filtration (see Theorem 2.4). In particular, we provide an example of a local prime noetherian PI ring for which the Jacobson radical does not satisfy the strong AR property. This is given in Section 4 where the reader may find further applications of the main theorem.

\section{Proof of Theorem 1.1}

Since we are also interested in $\mathfrak{m}$-adic filtrations of local rings as well as ascending filtrations, we will prove our main result for $\mathbb{Z}$-filtrations. First we review some basic facts about filtrations from [3, Chapter 6] and [4].

Suppose that $A$ is a $k$-algebra. For the purposes of this paper a filtration (or, more strictly, an exhaustive separated finite $\mathbb{Z}$-filtration) of $A$ is an ascending chain of subspaces $\Gamma=\left\{\Gamma_{i} \subseteq \Gamma_{i+1} \mid i \in \mathbb{Z}\right\}$ of $A$, satisfying:

(1) $1 \in \Gamma_{0}$ and $\Gamma_{i} \Gamma_{j} \subseteq \Gamma_{i+j}$ for all $i, j \in \mathbb{Z}$;

(2) $\Gamma$ is finite in the sense that $\operatorname{dim} \Gamma_{i} / \Gamma_{i-1}<\infty$ for all $i \in \mathbb{Z}$;

(3) $\Gamma$ is exhaustive in the sense that $A=\bigcup_{i \in \mathbb{Z}} \Gamma_{i}$ and separated in the sense that $\bigcap_{i \in \mathbb{Z}} \Gamma_{i}=0$.

The Rees ring associated to $\Gamma$ is defined to be Rees $A=\operatorname{Rees}_{\Gamma} A=\bigoplus_{i \in \mathbb{Z}} \Gamma_{i}$ and the associated graded ring is $\operatorname{gr} A=\operatorname{gr}_{\Gamma} A=\bigoplus_{i \in \mathbb{Z}} \Gamma_{i} / \Gamma_{i-1}$. Write $J(A)$ for the Jacobson radical of $A$. Following 4, a filtered algebra $A$ is called (left) Zariskian if

(Zar1) $\Gamma_{-1} \subseteq J\left(\Gamma_{0}\right)$;

(Zar2) $\operatorname{Rees}_{F} A$ is (left) noetherian.

Fix a filtration $\Gamma$ of the algebra $A$ and a left $A$-module $M$. The concept of a $\mathbb{Z}$-filtration (again, finite, exhaustive and separated) $\Lambda=\left\{\Lambda_{i}: i \in \mathbb{Z}\right\}$ of $M$ is defined analogously; one simply replaces (1) in the above definition by

(1') $\Gamma_{i} \Lambda_{j} \subseteq \Lambda_{i+j}$ for all $i, j \in \mathbb{Z}$.

Corresponding to this filtration one has the Rees module $\operatorname{Rees}_{\Lambda} M=\bigoplus \Lambda_{i}$ and associated graded module $\operatorname{gr}_{\Lambda} M=\bigoplus \Lambda_{i} / \Lambda_{i-1}$. We say that $\Lambda$ is a good filtration if there exist $\left\{m_{i} \in \Lambda_{d_{i}}: 1 \leq i \leq r<\infty\right\}$ such that $\Lambda_{n}=\sum_{i=1}^{r} \Gamma_{n-d_{i}} m_{i}$ for all $n \in \mathbb{Z}$. Two filtrations $\Lambda$ and $\Lambda^{\prime}$ of $M$ are equivalent, written $\Lambda \sim \Lambda^{\prime}$, if there is an integer $q$ such that $\Lambda_{i} \subset \Lambda_{i+q}^{\prime}$ and $\Lambda_{i}^{\prime} \subset \Lambda_{i+q}$ for all $i \in \mathbb{Z}$.

The Hilbert function of $M$ with respect to $\Lambda$ is defined to be

$$
H_{M, \Lambda}(n)=\operatorname{dim} \Lambda_{n} / \Lambda_{-n}, \quad \text { for all } n \in \mathbb{Z} .
$$


Let $H$ and $H^{\prime}$ be two (Hilbert) functions $\mathbb{N} \rightarrow \mathbb{N}$. We say that the growth of $H$ is at most the growth of $H^{\prime}$, and write $H \leq H^{\prime}$, if there is an integer $q$ such that $H(n) \leq H^{\prime}(n+q)$ for all $n \gg 0$. We say $H$ and $H^{\prime}$ are equivalent, written $H \sim H^{\prime}$, if both $H \leq H^{\prime}$ and $H^{\prime} \leq H$ hold.

Since we require filtrations to be separated, they need not induce filtrations on factor modules. However, for Zariskian filtrations this is not a problem:

Lemma 2.1. Suppose that $\Gamma=\left\{\Gamma_{i}\right\}$ is a filtration of an algebra $A$ and let $M$ be a left A-module with a good filtration $\Lambda$.

(1) If $\Lambda^{\prime}$ is another filtration of $M$, there exists an integer $q$ such that $\Lambda_{i} \subset \Lambda_{i+q}^{\prime}$ for all $i$. Thus, if $\Lambda^{\prime}$ is good, then $\Lambda$ and $\Lambda^{\prime}$ are equivalent.

(2) If $\Lambda^{\prime}$ and $\Lambda^{\prime \prime}$ are equivalent filtrations of $M$, then $H_{M, \Lambda^{\prime}}$ and $H_{M, \Lambda^{\prime \prime}}$ are equivalent.

(3) Assume that $\Gamma$ is left Zariskian. If $N$ is a submodule of $M$, then $\Lambda$ induces good filtrations on $N$ and $M / N$. In particular, $\Gamma$ induces a left Zariskian filtration on every factor ring of $A$.

Proof. (1) and (2) follow from the definitions while (3) follows from [4, Theorem 3.3] or [5] Theorem II.2.1.2].

The key observation in this paper is given by the next proposition. Since our main theorem will come in three slightly different forms, this result will also have three slightly different cases.

Proposition 2.2. Let $A$ be an algebra with a filtration $\Gamma$ such that the growth of $H_{A, \Gamma}$ is subexponential. Let $M$ be an A-bimodule such that the left module ${ }_{A} M$ is free of rank $s$ and the right module $M_{A}$ is free of rank $t$. Suppose that there exists a filtration $\Lambda$ on $M$ such that $\Lambda$ is a good filtration of ${ }_{A} M$ and a filtration of $M_{A}$.

(1) If $\Lambda$ is also a good filtration of $M_{A}$, then $t=s$.

(2) If $\Gamma_{n}=0$ for $n \ll 0$, then $s \geq t$.

(3) If $\Gamma_{n}=A$ for $n \gg 0$, then $s \leq t$.

Proof. The beginning of the proof is the same in all three cases. Note that ${ }_{A} A$ has a good filtration, simply because $\Gamma_{i}=\Gamma_{i} 1$ for all $i$. Thus ${ }_{A} A^{(s)}$ also has a good filtration and so this induces a good filtration $\Lambda^{\prime}$ on $M$ such that $\Lambda_{i}^{\prime} \cong \Gamma_{i}^{(s)}$ for all $i$. By Lemma [2.1(1), there exists $q_{1}$ such that $\Lambda_{i} \subseteq \Lambda_{i+q_{1}}^{\prime}$ for all $i$.

Similarly, the right $A$-module structure provides an induced good filtration $\Lambda^{\prime \prime}$ of $M_{A}$ such that $\Lambda_{i}^{\prime \prime} \cong \Gamma_{i}^{(t)}$ for all $i$. By Lemma 2.1(1), there exists $q_{2}$ such that $\Lambda_{i}^{\prime \prime} \subseteq \Lambda_{i+q_{2}}$ for all $i$. Hence, for $p=q_{1}+q_{2}$,

$$
\Lambda_{i}^{\prime \prime} \subseteq \Lambda_{i+p}^{\prime} \quad \text { for all } i \in \mathbb{Z} .
$$

We now consider the three cases separately. Under assumption (2), we see that $\Lambda_{i}^{\prime \prime} / \Lambda_{-i}^{\prime \prime}=\Lambda_{i}^{\prime \prime} \hookrightarrow \Lambda_{i+p}^{\prime}=\Lambda_{i+p}^{\prime} / \Lambda_{-(i+p)}^{\prime}$ for $i \gg 0$. Thus, $H_{M, \Lambda^{\prime \prime}}(n) \leq H_{M, \Lambda^{\prime}}(n+p)$. But, by construction, $H_{M, \Lambda^{\prime}}=s H_{A, \Gamma}$ and $H_{M, \Lambda^{\prime \prime}}=t H_{A, \Gamma}$ and so

$$
H_{A, \Gamma}(n) \leq \frac{s}{t} H_{A, \Gamma}(n+p) \quad \text { for all } n \in \mathbb{N} .
$$

Since $H_{A, \Gamma}(n)$ grows subexponentially this forces $t \leq s$.

Under assumption (3) we have $\Lambda_{i+p}^{\prime \prime} / \Lambda_{-(i+p)}^{\prime \prime}=M / \Lambda_{-i-p}^{\prime \prime} \rightarrow M / \Lambda_{-i}^{\prime}=\Lambda_{i}^{\prime} / \Lambda_{-i}^{\prime}$ for $i \gg 0$. Thus, $H_{M, \Lambda^{\prime}}(i) \leq H_{M, \Lambda^{\prime \prime}}(i+p)$ and repeating the analysis of the last paragraph shows that $s \leq t$. 
Finally, assume that $\Lambda$ is a good filtration of $M_{A}$. In this case, $\Lambda^{\prime \prime} \sim \Lambda$ and so $\Lambda^{\prime \prime} \sim \Lambda^{\prime}$. Thus there exists $p$ such that $\Lambda_{i}^{\prime} \subseteq \Lambda_{i+p}^{\prime \prime} \subseteq \Lambda_{i+2 p}^{\prime}$. Now a minor variant of the penultimate paragraph shows that $t \leq s$ and hence, by symmetry, that $s=t$.

Theorem 2.4. Suppose that $R$ is an algebra with ideals $I$ and $J_{1} \subset J_{2}$ such that $J_{2} / J_{1}$ is free of rank $s$ as a left $R / I$-module and free of rank $t$ as a right $R / I$ module. If $s<t$, then there is no filtration of $R$ such that $R$ is both left and right Zariskian.

Proof. Let $\widetilde{\Gamma}$ be a left and right Zariskian filtration of $R$. Then Lemma 2.1 3 ) implies that the induced filtration $\Gamma$ on $A=R / I$ is left and right Zariskian and so $\operatorname{gr}_{\Gamma} A$ is left (and right) noetherian. By [12, Remark after 1.2] the growth of $H_{A, \Gamma}$ is subexponential. By Lemma 2.1 3), $\Gamma$ induces a good filtration on $J_{2} / J_{1}$ as a left and a right $A$-module, which contradicts Proposition 2.2(1).

A curious feature of this result is that $R$ could be left or right Zariskian; it just cannot be both (see Corollary 4.7). However, with a little more information one can determine which side goes wrong.

Theorem 2.5. Suppose that $R$ is an algebra with ideals $I$ and $J_{1} \subset J_{2}$ such that $J_{2} / J_{1}$ is free of rank $s$ as a left $R / I$-module and free of rank $t$ as a right $R / I$-module. Assume that $s<t$ and that $\Gamma$ is a filtration of $R$.

(1) If $\Gamma_{i}=0$ for $i \ll 0$, then $\Gamma$ is not left Zariskian.

(2) If $\Gamma_{i}=A$ for $i \gg 0$, then $\Gamma$ is not right Zariskian.

Proof. For (1), repeat the proof of Theorem 2.4, but with Proposition 2.2(1) replaced by Proposition 2.2(2). For (2), use the proof of Theorem 2.4, applied to the opposite ring $R^{\mathrm{op}}$, with Proposition 2.2(1) replaced by Proposition 2.2(3).

We are now ready to prove Theorem 1.1 from the Introduction.

Proof of Theorem 1.1. It is easy to see that an $\mathbb{N}$-filtration $\Gamma$ of a ring $R$ is left Zariskian if and only if $\operatorname{gr}_{\Gamma}$ is left noetherian (see, for example, 5, Proposition II.1.2.3]). Thus, Theorem 1.1] is a special case of Theorem 2.5(1).

The analogue of Theorem 1.1 for complete local rings also holds with much the same proof. To state the result, we need some definitions. Let $R$ be a semilocal algebra with Jacobson radical $J(R)=\mathfrak{m}$ and assume that $\operatorname{dim}_{k} R / \mathfrak{m}<\infty$. A filtration $\Gamma$ of $R$ is called a weak adic filtration if it satisfies $\Gamma_{n}=A$ for all $n \geq 0$ and $\Gamma_{-1} \subseteq \mathfrak{m}$. (We still require the filtration to be finite, separated and exhaustive.)

Corollary 2.6. Let $R$ be a complete semilocal right noetherian algebra with Jacobson radical $\mathfrak{m}$. Suppose that $R$ has ideals $I$ and $J_{1} \subset J_{2}$ such that $J_{2} / J_{1}$ is free of rank $s$ as a left $R / I$-module and free of rank $t$ as a right $R / I$-module. If $s<t$, then there is no weak adic filtration $\Gamma$ such that $\operatorname{gr}_{\Gamma} R$ is right noetherian.

Proof. Suppose that such a filtration $\Gamma$ exists. By [2, Corollary 1.2] the filtration $\Gamma$ is also complete (in the natural sense that Cauchy sequences modulo the $\Gamma_{-i}$ should converge - see [5, Definition I.3.3.2]). Thus [5, Proposition II.2.2.1] implies that the filtration is right Zariskian and the result follows Theorem [2.5)(2). 
An analogue of this corollary also holds for noncomplete rings, although the result is less pleasant since one cannot now assume that separated filtrations induce separated filtrations on factor modules. The result becomes the following: Suppose that $R$ satisfies the hypotheses of Corollary 2.6 but that $R$ is not complete. Let $\Gamma$ be a weak adic filtration of $R$ that induces both a filtration of $R / I$ and a good right filtration of $J_{2} / J_{1}$. If $s<t$, then $\operatorname{gr}_{\Gamma} R$ is not right noetherian.

\section{A PARTial GENERALizAtion}

Although the results of the last section are sufficient for our examples, one can give a version of Theorem 1.1 that works without the assumption that $J_{2} / J_{1}$ be free, but at the expense of of assuming that $R / I$ be a prime Goldie ring. We prove this in this section. Let $A$ be a prime left Goldie ring with simple artinian ring of fractions $Q(A)$. If $M$ is a left $A$-module, then the Goldie rank of $M$ is defined to be the length of $Q(A) \otimes_{A} M$ and written $\operatorname{Grank}(M)$.

Lemma 3.1. Suppose that $A$ is a prime Goldie ring with a left noetherian $\mathbb{N}$ filtration $\Gamma$. Let $M$ be an A-bimodule with a filtration $\Lambda$ that is a good filtration of ${ }_{A} M$ and a filtration of $M_{A}$. If ${ }_{A} M$ is torsion, then so is $M_{A}$.

Proof. Assume that $M_{A}$ is not torsion. Replacing $M$ by $M^{(n)}$, for some $n$, we may assume that $A_{A} \subseteq M_{A}$. Thus, Lemma 2.11) implies that there exists $q \geq 0$ such that

$$
\operatorname{dim} \Gamma_{n} \leq \operatorname{dim} \Lambda_{n+q} \cap A \leq \operatorname{dim} \Lambda_{n+q}
$$

for all $n$. Since $\Gamma_{n}=\Lambda_{n}=0$ for $n \ll 0$, this implies that $H_{M, \Lambda} \geq H_{A, \Gamma}$.

Now consider ${ }_{A} M$. Since $\Lambda$ is a good filtration, ${ }_{A} M$ is finitely generated. We claim, for all $p>0$, that $H_{M, \Lambda} \leq \frac{1}{p} H_{A, \Gamma}$. Once this has been proved, then the last paragraph implies, for some $x>0$, that $H_{A, \Gamma}(n) \leq \frac{1}{p} H_{A, \Gamma}(n+x)$. This contradicts the fact that $H_{A, \Gamma}$ grows subexponentially [12] Remark after 1.2] and proves the lemma. In order to prove the claim we ignore the right-hand structure of $M$ and so, by induction, it suffices to prove it for a cyclic module $M=A / I$. Since ${ }_{A} M$ is torsion and $A$ is Goldie, $I$ contains a regular element, $a$ say, of $A$. We will still write $\Gamma$ for the good filtration on any subfactor of ${ }_{A} A$ induced from $\Gamma$. Now, for any $n, M$ is a homomorphic image of $L(i)=A a^{i-1} / A a^{i} \cong A / A a$ and so $H_{M, \Lambda} \leq H_{L(i), \Gamma}$. Since Hilbert series are additive on short exact sequences, this implies that $p H_{M, \Lambda} \leq H_{A / A a^{p}, \Gamma} \leq H_{A, \Gamma}$, for any $p \in \mathbb{N}$.

Theorem 3.2. Suppose that $I$ and $J_{1} \subset J_{2}$ are ideals of an algebra $R$ such that $A=R / I$ is a prime Goldie ring. Assume that $J_{2} / J_{1}$ has Goldie rank $s$ as a left $A$-module and Goldie rankt as a right $A$-module, for some $s<t$. Then there is no $\mathbb{N}$-filtration $\Gamma^{\prime}$ of $R$ such that $\operatorname{gr}_{\Gamma^{\prime}} R$ is left noetherian.

Proof. Suppose that such a filtration $\Gamma^{\prime}$ exists. Let $\Gamma$ be the induced filtration on $A$ and $\Lambda$ the induced filtration on $M=J_{2} / J_{1}$. Then $\Lambda$ is a good left filtration and so ${ }_{A} M$ is finitely generated. The torsion submodule $T$ of $M_{A}$ is an $A$-bimodule and so we may pass to $M / T$ without affecting the hypotheses (although $s$ may decrease). By Lemma $3.1{ }_{A} M$ is also torsion-free. If $\operatorname{Grank}(A)=u$, replace $M$ by $M^{(u)}$; thus $A_{A}^{(t)} \cong X \subseteq M_{A}$ and ${ }_{A} M \subseteq Y \cong{ }_{A} A^{(s)}$. Let $\Theta$, respectively $\Phi$, be the filtrations of $X$ and $Y$ induced from $\Gamma$. Since $\Theta$ equals the direct sum $\Gamma^{(t)}$ of $t$ copies of $\Gamma$, certainly $H_{X, \Theta}=t H_{A, \Gamma}$. Similarly, $H_{Y, \Phi}=s H_{A, \Gamma}$. 
Since $\Lambda$ is a good left filtration and $\Theta$ is a good right filtration, by Lemma 2.1 1 ) there exists $q \geq 0$ such that

$$
\operatorname{dim} \Theta_{n} \leq \operatorname{dim}\left(X \cap \Lambda_{n+q}\right) \leq \operatorname{dim} \Lambda_{n+q}
$$

and

$$
\operatorname{dim} \Lambda_{n} \leq \operatorname{dim}\left(M \cap \Phi_{n+q}\right) \leq \operatorname{dim} \Phi_{n+q},
$$

for all $n \gg 0$. Hence,

$$
t H_{A, \Gamma}(n)=H_{X, \Theta}(n) \leq H_{Y, \Phi}(n+2 q)=s H_{A, \Gamma}(n+2 q),
$$

for all $n \gg 0$. Since $H_{A, \Gamma}$ grows subexponentially, this forces $t \leq s$.

With a rather more complicated argument one can prove an analogous version of Theorem 2.4 using Goldie ranks. However, we do not know how to prove the analogous version of Theorem 2.5 (2) or Corollary 2.6 without extra hypotheses.

\section{EXAMPLES}

In this section we use the results of Section 2 to provide examples of rings without noetherian associated graded rings.

Example 4.1. Let

$$
S=\left\{\left(\begin{array}{cc}
f(x) & g(x) \\
0 & f\left(x^{2}\right)
\end{array}\right): f, g \in k[x]\right\}+y M_{2}(k[x, y]) \subset M_{2}(k[x, y]) .
$$

Then $S$ is an affine noetherian prime PI algebra without any left noetherian finite $\mathbb{N}$-filtrations.

Proof. The diagonal matrices of the form

$$
\left(\begin{array}{cc}
f(x, y) & 0 \\
0 & f\left(x^{2}, y\right)
\end{array}\right): f(x, y) \in k[x, y]
$$

clearly form a subring $C$ of $S$ isomorphic to $k[x, y]$. Since $S$ is finitely generated as a left or right $C$-module, it follows that $S$ is a finitely generated noetherian PI algebra. It is prime since it contains a nonzero ideal of the prime ring $M_{2}(k[x, y])$.

Suppose that $A \rightarrow B$ is a surjective homomorphism of algebras and that $B$ does not admit a left noetherian $\mathbb{N}$-filtration. Then an immediate consequence of Lemma 2.1(3) is that $A$ also has no such filtration. Thus, it suffices to prove the final assertion for a factor ring, and we use

$$
R=S / y M_{2}(k[x, y]) \cong\left\{\left(\begin{array}{cc}
f(x) & g(x) \\
0 & f\left(x^{2}\right)
\end{array}\right): f, g \in k[x]\right\} \subset M_{2}(k[x]) .
$$

Notice that this is the ring from (1.2). The nilradical $N=N(R)$ is just the set of strictly upper triangular matrices. It is routine to check that $N$ is a free left $R / N$-module of rank one but a free right $R / N$-module of rank two. Hence, by Theorem 1.1, neither $R$ nor $S$ can have a left noetherian finite $\mathbb{N}$-filtration.

Remark 4.3. The ring $R$ from (4.2) clearly has Gelfand-Kirillov dimension one. By inspecting the proof of Theorem 2.4, this shows that there is no finite filtration of $R$ that induces a good filtration of $N(R)$ as a left $R$-module.

Other examples of noetherian rings with no noetherian $\mathbb{N}$-filtration are given in [12. However, those examples require that the ring in question have infinite Gelfand-Kirillov dimension and this is impossible for affine PI algebras (see [8] Proposition 13.10.6]). 
It is readily checked that this ring $R$ does have a right noetherian, finite filtration (see Corollary 4.7). By slightly modifying the example one can get an example that "works" on both sides.

Example 4.4. Let

$$
T=\left\{\left(\begin{array}{ccc}
f(x) & g(x) & h(x) \\
0 & f\left(x^{2}\right) & l(x) \\
0 & 0 & f(x)
\end{array}\right): f(x), \ldots, l(x) \in k[x]\right\}+y M_{3}(k[x, y]) .
$$

Then $T$ is an affine noetherian prime PI algebra such that, for every $\mathbb{N}$-filtration, the associated graded ring is neither left nor right noetherian.

Proof. Notice that $T /\left(e_{13} T+e_{23} T\right) \cong R$, the ring from (4.2), and so $T$ has no left noetherian finite filtration. Since $T \cong T^{o p}$, the same is true on the right.

These examples can be easily modified into complete local rings and we give one example that is analogous to the ring $R$ from Example 4.1. Before giving the example, we need a definition. An ideal $I$ of a $\operatorname{ring} R$ is said to satisfy the strong $A R$ property if the associated Rees ring $\operatorname{Rees}_{I} R=\bigoplus_{j \geq 0} I^{j}$ is noetherian. The significance of this condition is that, if $I$ satisfies the strong AR property, then it also satisfies the usual Artin-Rees (AR) property; indeed this is the standard way of proving the latter condition in commutative algebra. The same idea has been useful in noncommutative algebra (see, for example, [10, §2] and [5]). Given that the Jacobson radical of a semi-local noetherian PI algebra is automatically AR (1) Theorems 3.1.13 and 7.2.5]), it is natural to ask if it is also strongly AR.

The next example shows that it does not. Notice that, although the ring in question is just a completion of the ring $R$ from Example 4.1 the Zariskian property fails on the opposite side.

Example 4.5. Let

$$
R=\left\{\left(\begin{array}{cc}
f(x) & g(x) \\
0 & f\left(x^{2}\right)
\end{array}\right): f, g \in k[[x]]\right\}+y M_{2}(k[[x, y]]) \subset M_{2}(k[[x, y]]) .
$$

Then $R$ is a prime noetherian complete local PI algebra over $k$. Moreover, $R$ has no weak adic filtration $\Gamma$ such that $\operatorname{gr}_{\Gamma} R$ is right noetherian. In particular, $J(R)$ does not satisfy the strong AR property.

Proof. The proof that $R$ is a prime noetherian PI algebra over $k$ is analogous to that of Example 4.1 and is left to the reader. Clearly $R$ is a complete local ring.

In order to complete the proof it suffices, by Corollary 2.6 to work in a factor ring and we choose

$$
R^{\prime}=R / y M_{2}(k[[x, y]])=\left\{\left(\begin{array}{cc}
f(x) & g(x) \\
0 & f\left(x^{2}\right)
\end{array}\right): f(x), g(x) \in k[[x]]\right\} .
$$

The nilradical $N$ of $R^{\prime}$ is the set of strictly upper triangular matrices and is free of rank 1 as a left $R^{\prime} / N$-module and free of rank 2 as a right $R^{\prime} / N$-module. Now apply Corollary 2.6

Finally we justify a remark made after Theorem 2.4 In that theorem it is possible to have a filtration that is Zariskian on either the left or the right. 
Corollary 4.7. Consider noetherian, PI algebras $R$ with ideals $I$ and $J_{1} \subseteq J_{2}$ such that $J_{2} / J_{1}$ is a free left $R / I$-module of rank one and a free right $R / I$-module of rank 2 .

Then, there exists an example $R_{1}$ of such a ring with a left Zariskian filtration $\Gamma_{1}$ and an example $R_{2}$ of such a ring with a right Zariskian filtration $\Gamma_{2}$.

Proof. The ring $R_{2}$ is just the ring $R$ from (1.2), and so it does satisfy the hypotheses of the first paragraph. Set

$$
\alpha=\left(\begin{array}{cc}
x & 0 \\
0 & x^{2}
\end{array}\right) \text { and } \beta=\left(\begin{array}{ll}
0 & 1 \\
0 & 0
\end{array}\right) .
$$

It is easy to see that $R$ is generated by $\alpha$ and $\beta$ and hence one has the standard $\mathbb{N}$ filtration $\Gamma_{0}=k, \Gamma_{1}=k+k \alpha+k \beta$ and $\Gamma_{n}=\Gamma_{1}^{n}$ for $n \geq 2$. Let $a$ and $b$ be the images of $\alpha$ and $\beta$ in $\operatorname{gr}_{\Gamma} R$. Then, $\operatorname{gr}_{\Gamma} R$ is generated by $a$ and $b$ and satisfies the relations $a^{2} b=0=b^{2}$. Thus $\operatorname{gr}_{\Gamma} R$ is spanned by the elements $\left\{a^{n}, b a^{n}, a b a^{n}: n \geq 0\right\}$. As such, $\operatorname{gr}_{\Gamma} R$ is a finitely generated right $k[a]$-module, and so is right noetherian. As in the proof of Theorem 1.1 this suffices to prove that $\Gamma$ is right Zariskian.

For $R_{1}$ we take the ring $R^{\prime}$ from (4.6). We define $\alpha$ and $\beta$ by (4.8) and observe that $\mathfrak{m}=J\left(R^{\prime}\right)=R^{\prime} \alpha+R^{\prime} \beta$. Now use the $\mathfrak{m}$-adic filtration $\Gamma$ defined by $\Gamma_{i}=R^{\prime}$ if $i \geq 0$ but $\Gamma_{i}=\mathfrak{m}^{-i}$ if $i \leq 0$. Then $\beta \alpha=\alpha^{2} \beta \in \mathfrak{m}^{3}$. Hence in the associated graded ring one finds that the images of these elements satisfy $b a=0=b^{2}$. The argument of the last paragraph shows that $\operatorname{gr}_{\Gamma} R^{\prime}$ is left noetherian and hence, by [5, Proposition II.1.2.3], that $\Gamma$ is left Zariskian.

\section{A DUALIZING MOdUle}

As was remarked in the Introduction, if an affine noetherian PI algebra $R$ has a finite noetherian $\mathbb{N}$-filtration, then $R$ has a dualizing complex. Thus, one can ask whether the ideas of the last section can be used to provide examples of PI rings which do not have such a complex. This appears not to be the case; in this section we check that the ring $R$ from (1.2) does indeed have such a complex.

One advantage of this example is that we can work with modules rather than complexes, and so we define an $(R, R)$-bimodule $D$ to be a dualizing module if (i) $D$ is finitely generated and of finite injective dimension on both sides, (ii) the natural maps $R \rightarrow \operatorname{End}\left(D_{R}\right)$ and $R^{\text {op }} \rightarrow \operatorname{End}\left({ }_{R} D\right)$ are isomorphisms, and (iii) $\operatorname{Ext}_{R}^{i}\left(D_{R}, D_{R}\right)=\operatorname{Ext}_{R}^{i}\left({ }_{R} D,_{R} D\right)=0$ for all $i>0$. A dualizing module viewed as a complex is a dualizing complex in the sense of Yekutieli [14].

The way we find a dualizing module for the ring $R$ from (1.2) is through the following observation: Identify $C=k[x]$ with the diagonal matrices in $R$ and set

$$
D_{1}=\operatorname{Hom}_{C}(C R, C) \quad \text { and } \quad D_{2}=\operatorname{Hom}_{C}\left(R_{C}, C\right) .
$$

Thus, $D_{1}$ is an $(R, C)$-bimodule and $D_{2}$ is a $(C, R)$-bimodule. The key to our construction is the following easy lemma.

Lemma 5.2. Let $R \supseteq C$ be rings such that $R$ is a finitely generated projective $C$ module on both sides and $C$ is a commutative noetherian algebra of finite injective dimension. Define modules $D_{i}$ by (5.1). Suppose that ${ }_{C} D_{1}$ and $\left(D_{2}\right)_{D}$ are projective modules and that one has ring isomorphisms $\operatorname{End}_{R}\left(D_{1}\right) \cong R^{\text {op }}$ and $\operatorname{End}_{R}\left(D_{2}\right) \cong R$ through which $D_{1} \cong D_{2}$ as $R$-bimodules. Then, $D=D_{1}$ is a dualizing module for $R$. 
Proof. It is clear that $C$ is a dualizing module for itself. By [9, Theorem 11.66] one has natural isomorphisms

$$
\operatorname{Ext}_{R}^{i}\left({ }_{R} N,{ }_{R} D_{1}\right)=\operatorname{Ext}_{R}^{i}\left({ }_{R} N, \operatorname{Hom}_{C}\left({ }_{C} R, C\right)\right) \cong \operatorname{Ext}_{C}^{i}\left({ }_{C} N, C\right),
$$

for any finitely generated left $R$-module $N$. This implies that the injective dimension of ${ }_{R} D_{1}$ is bounded by the injective dimension of ${ }_{C} C$. Since ${ }_{C} D_{1}$ is projective, (5.3) implies that $\operatorname{Ext}^{i}\left({ }_{R} D_{1}, R D_{1}\right)=0$ for all $i>0$. Similar assertions hold for $\left(D_{2}\right)_{R}$. It follows from [11, Theorem 3.5] that ${ }_{R} D_{1}$ and $\left(D_{2}\right)_{R}$ are finitely generated. Therefore $D=D_{1}$ is a dualizing module.

Proposition 5.4. Let $R$ be the ring defined by (1.2) and define modules $D_{i}$ by (5.1). Then, $D=D_{1}$ is a dualizing module for $R$.

Proof. We check that the hypotheses of the lemma are satisfied for $C=k[x]$. Since $R$ is a free left $k[x]$-module of rank two and a free right $k[x]$-module of rank three, the proof is a routine computation which we will only outline. One first checks that, under the natural module structures,

$$
D_{1} \cong \frac{R \oplus R}{R\left(x e_{12}, e_{12}\right)} \quad \text { and } \quad D_{2} \cong R / e_{12} R,
$$

where $e_{12}=\left(\begin{array}{ll}0 & 1 \\ 0 & 0\end{array}\right)$ is the matrix unit. Since $e_{12}$ is one of the basis elements of $R$ as a left or right $C$-module, certainly ${ }_{C} D_{1}$ and $\left(D_{2}\right)_{C}$ are free. It follows that

$$
\operatorname{End}_{R}\left(D_{2}\right) \cong I\left(e_{12} R\right) / e_{12} R \quad \text { where } \quad I(a R)=\{\theta \in R: \theta a R \subseteq a R\} .
$$

Computing this out one finds that

$$
\begin{aligned}
\operatorname{End}_{R}\left(D_{2}\right) & \cong\left\{\left(\begin{array}{cc}
f\left(x^{2}\right) & g(x) \\
0 & f\left(x^{4}\right)
\end{array}\right): f, g \in k[x]\right\} / e_{12} k\left[x^{2}\right] \\
& \cong\left\{\left(\begin{array}{cc}
f\left(x^{2}\right) & g\left(x^{2}\right) \\
0 & f\left(x^{4}\right)
\end{array}\right): f, g \in k[x]\right\} \cong R .
\end{aligned}
$$

Finally, under this isomorphism $E=\operatorname{End}_{R}\left(D_{2}\right) \cong R$ one finds that the modules ${ }_{E} D_{2}$ and ${ }_{R} D_{1}$ become isomorphic, as is required to prove the proposition.

It would be interesting to know whether this proof can be extended to work for any ring $S$ that is finitely generated as a module over a commutative subring. The fact that the present proof depends upon the "lucky" isomorphism $\operatorname{End}_{R}\left(D_{2}\right) \cong R$ makes this seem unlikely. By using ideas from [13, it can at least be extended to Hopf algebras finitely generated as modules over commutative subalgebras.

\section{ACKNOWLEDGMENT}

The authors thank Quanshui Wu for several conversations on the subject.

\section{ADDED IN PROOF}

One of the questions raised in the introduction (whether noetherian affine PI Hopf algebras must have finite injective dimension) has since been answered in the positive. See Q. Wu and J. J. Zhang, Noetherian PI Hopf algebras are Gorenstein, Trans. Amer. Math. Soc. 355 (2003), 1043-1066. 


\section{REFERENCES}

1. A. V. Jategaonkar, Localization in Noetherian Rings, CUP, Cambridge, 1986. MR 88c:16005

2. A. V. Jategaonkar, Morita duality and noetherian rings, J. Algebra, 69 (1981), 358-371. MR 82j:16036

3. G. R. Krause and T. H. Lenagan, Growth of Algebras and Gelfand-Kirillov Dimension (Revised edition), Graduate Studies in Mathematics, 22, Amer. Math. Soc., Providence, RI, 2000. MR 2000j: 16035

4. H. Li and F. Van Oystaeyen, Zariskian filtrations. Comm. in Algebra, 17 (1989), 2945-2970. MR 90m:16004

5. H. Li and F. Van Oystaeyen, Zariskian Filtrations. K-Monographs in Mathematics, Kluwer Academic Publishers, Dordrecht, 1996. MR 97m:16083

6. M. Lorenz, On Gelfand-Kirillov dimension and related topics, J. Algebra, 118 (1988), 423437. MR 89m:16004

7. M. Lorenz, Gelfand-Kirillov Dimension and Poincaré Series, Cuadernos de Algebra, No.7, Universidad de Grenada, Grenada, 1988.

8. J. C. McConnell and J. C. Robson, Noncommutative Noetherian Rings, Wiley, Chichester, 1987. MR 89j:16023

9. J. J. Rotman, An Introduction to Homological Algebra, Academic Press, New York, 1979. MR 80k:18001

10. J. T. Stafford and N. R. Wallach, The restriction of admissible modules to parabolic subalgebras, Trans. Amer. Math. Soc., 272 (1982), 333-350. MR 83h:17007

11. J. T. Stafford and J. J. Zhang, Homological properties of (graded) noetherian PI rings, J. Algebra, 168 (1994), 988-1026. MR 95h:16030

12. D. R. Stephenson and J. J. Zhang, Growth of graded noetherian rings, Proc. Amer. Math. Soc., 125 (1997), 1593-1605. MR 97g:16033

13. Q. Wu and J. J. Zhang, Homological identities for noncommutative rings, J. Algebra, 242 (2001), 516-535. MR 2002k:16011

14. A. Yekutieli, Dualizing complexes over noncommutative graded algebras, J. Algebra, 153 (1992), 41-84. MR 94a:16077

15. A. Yekutieli and J. J. Zhang, Rings with Auslander dualizing complexes, J. Algebra, 213 (1999), 1-51. MR 2000f:16012

Department of Mathematics, University of Michigan, Ann Arbor, Michigan 481091109

E-mail address: jts@umich.edu

Department of Mathematics, Box 354350, University of Washington, Seattle, WashINGTON 98195

E-mail address: zhang@math.washington.edu 